\title{
SNR Estimation for Multi-dimensional Cognitive Receiver under Correlated Channel/Noise
}

\author{
Shree Krishna Sharma, Student Member, IEEE, Symeon Chatzinotas, Member, IEEE \\ and Björn Ottersten, Fellow, IEEE
}

\begin{abstract}
In addition to Spectrum Sensing (SS) capability required by a Cognitive Radio (CR), Signal to Noise Ratio (SNR) estimation of the primary signals at the $C R$ receiver is crucial in order to adapt its coverage area dynamically using underlay techniques. In practical scenarios, channel and noise may be correlated due to various reasons and SNR estimation techniques with the assumption of white noise and uncorrelated channel may not be suitable for estimating the primary SNR. In this paper, firstly, we study the performance of different eigenvaluebased SS techniques in the presence of channel or/and noise correlation. Secondly, we carry out detailed theoretical analysis of the signal plus noise hypothesis to derive the asymptotic eigenvalue probability distribution function (a.e.p.d.f.) of the received signal's covariance matrix under the following two cases: (i) correlated channel and white noise, and (ii) correlated channel and correlated noise, which is the main contribution of this paper. Finally, an SNR estimation technique based on the derived a.e.p.d.f is proposed in the presence of channel/noise correlation and its performance is evaluated in terms of normalized Mean Square Error (MSE). It is shown that the PU SNR can be reliably estimated when the CR sensing module is aware of the channel/noise correlation.
\end{abstract}

Index Terms-Cognitive Radio, Spectrum Sensing (SS), Signal to Noise Ratio (SNR) Estimation, Channel/Noise Correlation, Random Matrix Theory (RMT))

\section{INTRODUCTION}

D URING the last decade, the demand for high speed wireless connections has constantly been increasing due to the proliferation of multimedia services. However, the available frequency resources are becoming scarce due to spectrum segmentation and dedicated frequency allocation of standardized wireless systems. In this context, Cognitive Radio (CR) is considered a promising candidate for enhancing the spectrum efficiency of communication systems because it is aware of its operating environments and can adjust its parameters dynamically $[1,2]$. For practical implementation of a CR, it is extremely important to explore efficient Spectrum Sensing (SS) techniques which can detect the presence of signals reliably. In addition to SS capability required by the CR, estimating the Signal to Noise Ratio (SNR) of the PU signals accurately is crucial in order to allow the spectral coexistence of primary and secondary systems using underlay techniques.

Several SS techniques have been proposed in the literature for CR based systems [3] and they have different operational requirements, advantages and disadvantages. The three main

The authors are with the Interdisciplinary Centre for Security, Reliability and Trust (SnT), University of Luxembourg (http://www.securityandtrust.lu) email: \{shree.sharma, symeon.chatzinotas, bjorn.ottersten\}@uni.lu.
SS techniques for sensing the presence of a Primary User (PU) that appear in the literature are matched filter detection, Energy Detection (ED) and cyclostationary feature detection [1]. Matched filter and cyclostationary methods require the prior knowledge about the PU signal to decide about the presence or absence of on-going primary transmissions. The ED technique does not require any knowledge of PU signals but the performance of this method relies on the accurate knowledge of the noise power [4]. Furthermore, several diversity enhancing techniques such as multi-antenna, cooperative and oversampled techniques have been introduced in the literature to enhance SS efficiency in wireless fading channels [5-7]. Most of aforementioned methods involve decision statistics calculated based on the eigenvalue distribution of the received signal's covariance matrix and use recent results from Random Matrix Theory (RMT) [8,9]. The eigenvalue-based SS technique does not require any prior information of the PU's signal and it outperforms ED techniques, especially in the presence of noise covariance uncertainty [5].

\section{A. Motivation and Contributions}

In the context of eigenvalue-based SS techniques, the following three practical scenarios have been considered in the literature with respect to the receive dimensions of the CR node: (i) Cooperative SS scenario [7], (ii) Multiantenna SS scenario [5,10], and (iii) Oversampled SS scenario [5]. In this paper, we consider a multi-dimensional framework which can be applicable to all these scenarios. The main problems that may arise in these practical scenarios are that the channel may be correlated across the receive dimensions due to insufficient scattering in the propagation path [11] and mutual coupling between antennas [12] and the noise may also be correlated due to imperfections in filtering and oversampling in the receiver [5]. Due to physical constraints of antenna spacing in Multiple Input Multiple Output (MIMO) systems and lack of rich scattering, it is not always feasible to consider independent MIMO channels in practice. In this context, it has been shown in [13] that spatial correlation may degrade the performance of wireless systems. Under these conditions, the channel matrix may become ill-conditioned and the maximum to the minimum eigenvalue ratio, called Standard Condition Number ( $\mathrm{SCN})$, can be used to describe the ill-conditioning of a wireless channel [14]. Since the performance of eigenvaluebased techniques is affected by the SCN of the channel covariance matrix, we investigate the behavior of RMT based techniques in the presence of channel correlation as well as in 
the presence of both channel/noise correlation. In this context, we use a SCN-based approach to model the correlation level and study the effect of channel/noise correlation on multidimensional SS techniques.

Furthermore, most SS related works focus on a dual hypothesis test to decide the presence or absence of primary on-going transmissions. If the CR is able to estimate the SNR of the primary signals, defined as the ratio of the received primary power to the noise power at the $\mathrm{CR}$ receiver, it can dynamically adapt its coverage area using underlay techniques. In order to implement underlay techniques such as power control at the CR, we need to know the SNR threshold required for the power control algorithm. Furthermore, to calculate the SNR threshold, we need to calculate the distance between the Primary Receiver (PR) and the CR. We consider Effective Isotropic Radiated Power (EIRP) of the Primary Transmitter (PT) as the cognition information at the CR and assume a Line of Sight (LoS) reciprocal channel. Based on EIRP limits of the PT and estimated received SNR of the primary signals, the spatial distance between the PT and the CR can be estimated and subsequently, based on this estimated distance, the secondary network can apply distancebased adaptive power control mechanism to adjust its coverage area. To clarify the above scheme, the following two scenarios can be considered. The first scenario assumes duplex mode of transmission for the PUs i.e., each user interchangeably transmits and receives over time. If we fix the SNR threshold based on estimated SNR over multiple time slots, we can also protect the weakest one assuming they have the same interference threshold [15]. The second scenario considers the simplex mode of transmission for the PUs and a short range wireless communication for both primary and secondary systems provided that interference levels from one system to another are at a similar level. In practice, it may be the case that a spectrum resource is completely left unused within a sufficiently large network coverage area. In this context, the optimal exploitation of spectrum holes depends on the maximally acceptable coverage area of secondary transmission while protecting the primary rate [9]. In such type of systems, it can be assumed that setting SNR threshold for the PT is a reasonable strategy for protecting the PR as well. In this context, based on the estimated PU SNR, suitable underlay techniques such as exclusion zone [16] can be applied.

In the context of CR networks, SNR estimation can be very useful in switching between underlay and interweave (SS) modes adaptively. In the SS only technique, the SUs are not allowed to access a particular PU channel when the channel is found to be occupied. In this scheme, the secondary network may have very low throughput specifically in heavily occupied spectrum regions. If the $\mathrm{CR}$ node has the capability of estimating the PU SNR along with its sensing ability, the SU can access the channel with full power in case of an idle channel and access the channel with controlled power in case of the occupied channel. Based on the link budget analysis and the interference constraint, proper SNR threshold can be determined to guarantee the protection of the PU rate. Subsequently, by comparing the estimated SNR with the SNR threshold, power control mechanism can be implemented at the CR to adjust its coverage area. More specifically, in the SS only techniques, the noise only hypothesis is decided if $\mathrm{SNR} \leq \lambda_{1}, \lambda_{1}$ being the decision threshold and the signal plus noise hypothesis is decided if SNR $>\lambda_{1}$. When we combine SS with the SNR estimation, we can introduce another threshold $\lambda_{2}$ under the signal plus noise hypothesis in the following way. If SNR $\leq \lambda_{2}$, then the CR can transmit in the same channel using the power control algorithm based on the interference threshold of the PU and the CR must stop its transmission when SNR $>\lambda_{2}$. Furthermore, the PU SNR knowledge provides channel quality information for the secondary system, which can be further used for implementing adaptive techniques such as adaptive bit loading, handoff algorithms and optimal soft value calculation for improving the performance of channel decoders [17]. Despite its important applications, only a few contributions in the literature address the SNR estimation problem in the context of a CR [18-21]. Furthermore, the SNR estimation techniques proposed under the assumption of the white noise and uncorrelated channel scenario may not perform well in the presence of channel/noise correlation. Taking the above into account, exploring efficient SNR estimation techniques in the presence of channel/noise correlation is an important research challenge.

In contrast to the application of the eigenvalue-based analysis for SS in most of the contributions [5-7], the focus of this paper is not on SS. The theoretical analysis carried out in this paper has been inspired by the multi-dimensional eigenvalue-based SS model [22] and it has been applied for SNR estimation application by carrying out analysis under signal plus noise hypothesis ${ }^{1}$. In our previous work [22], the effect of noise correlation on eigenvalue-based SS techniques has been studied and the a.e.p.d.f of the received signal's covariance matrix has been derived in the presence of correlated noise. In this paper, we focus on studying the SNR estimation problem in the presence of channel correlation and in the presence of both channel/noise correlation. For this purpose, we need the expressions for a.e.p.d.f. of the received signal's covariance matrix under above mentioned scenarios. However, these expressions are not available in the current literature including [22]. In this context, we derive the expressions for the a.e.p.d.f. of the received signal's covariance matrix in the presence of channel correlation and in the presence of both channel/noise correlation using RMT. This is the main contribution of this paper. Furthermore, we use these a.e.p.d.f. expressions in order to estimate the PU SNR in the presence of channel correlation and in the presence of both channel/noise correlation based on the maximum eigenvalue. Moreover, the performance of the proposed technique is evaluated in terms of normalized Mean Square Error (MSE).

\section{B. Structure and Notation}

This paper is structured as follows: Section II reviews in detail prior work related to SS and SNR estimation techniques in the presence of noise/channel correlation. Section III describes the considered signal models and further presents the causes of

\footnotetext{
${ }^{1}$ In this context, no methods for improving probability of detection in the presence of correlation have been included in this paper.
} 
channel and noise correlation in practical scenarios. Section IV reviews different eigenvalue-based blind SS techniques. Section $\mathrm{V}$ presents channel correlation analysis under signal plus noise hypothesis using RMT. Section VI provides the main analytical results for signal plus noise case in the presence of noise and/or channel correlation and further presents the PU SNR estimation method using the derived a.e.p.d.f. expressions. Section VII studies the performance of eigenvalue-based SS techniques in the presence of correlation and evaluates the performance of the proposed SNR estimation technique with numerical simulations. Section VIII concludes the paper. The appendix includes some preliminaries on random matrix transforms and proofs of the theorems.

Throughout this paper, boldface upper and lower case letters are used to denote matrices and vectors respectively, $\mathbb{E}[\cdot]$ denotes expectation, $\mathbb{C}$ denotes complex numbers, $(\cdot)^{T}$ and $(\cdot)^{H}$ denote the transpose and the conjugate transpose respectively, $(\cdot)^{*}$ represents the complex conjugate, $\mathbf{I}$ denotes the identity matrix, $(z)^{+}$denotes $\max (0, z), \mathbf{R}_{\mathbf{X}}$ represents the covariance matrix of $\mathbf{X}, \hat{\mathbf{R}}_{\mathbf{X}}$ represents the sample covariance of $\mathbf{X}, \mathcal{S}_{\mathbf{X}}$ represents the Stieltjes transform of $\mathbf{X}, \mathcal{R}_{\mathbf{X}}$ represents the $\mathrm{R}$ transform ${ }^{2}, \Sigma_{\mathbf{X}}$ represents the $\Sigma$ transform and $\eta_{\mathbf{X}}$ represents the $\eta$ transform [8].

\section{RELATED WORK}

RMT has been used in the literature in various applications such as modeling transmit/receive correlation in MIMO channels and multiuser MIMO fading [23,24]. It has also received considerable attention in the CR research community, specifically in the eigenvalue-based SS literature [5, 6, 13, 25]. The Maximum to Minimum Eigenvalue (MME) and Energy with Minimum Eigenvalue (EME) algorithms for SS have been proposed in [5] and approximate expressions for probability of a false alarm $\left(P_{f}\right)$ and probability of detection $\left(P_{d}\right)$ have been derived using RMT. The SS techniques based on MarchenkoPastur (MP) deterministic bounds, Tracy-Widom (TW) distribution and Tracy-Widom Curtiss (TWC) distribution have been proposed in [25], [5] and [26] respectively. Moreover, the approximate and exact distributions of the SCN of the received signal's covariance matrix have been used for SS purposes [6, 27, 28]. Among all existing techniques, SCN based methods are found to be preferable in presence of noise covariance uncertainty [6].

Recently, the distribution of the SCN of Wishart matrices has been considered in signal detection for a CR [14]. In this context, two types of condition numbers i.e., SCN and Demmel Condition Number (DCN) (the ratio of the matrix trace to the minimum eigenvalue) have been considered. In [14], a general framework for the Cumulative Distribution Function (CDF) of the SCN of different classes of Wishart matrices has been presented. In [29], the exact distribution of the DCN for random matrices with arbitrary dimensions has been presented. In [30], analytical expressions for the Probability Density Function (PDF) and Cumulative Distribution Function $(\mathrm{CDF})$ of the ratio of the largest eigenvalue to the trace of

\footnotetext{
${ }^{2}$ Readers should not confuse the $\mathrm{R}$ transform notation $\mathcal{R}$ with the covariance matrix notation $\mathbf{R}$.
}

complex Wishart matrices with arbitrary dimensions have been derived and the derived analytical results are used to find the decision threshold for a blind Generalized Likelihood Ratio Test (GLRT) detector. Furthermore, free probability theory, which is a valuable tool for describing the asymptotic behavior of multiple systems, has also received attention in the SS literature $[31,32]$.

In [18], an SNR estimation method has been proposed for ultra-wideband CR systems using computer simulations and this method is specific only for multiband orthogonal frequency division multiplexing-based systems. In [19], a pseudo bit error rate based SNR estimation has been proposed for the ED scheme. The contribution in [20] utilizes the priori knowledge of SNR, which can be obtained using estimation techniques, for realizing the adaptive SS techniques. Similarly in [21], a cooperative SS scheme has been proposed based on the SNR estimation and the energy combining method, where the combining weights for the received energies of cooperative sensors are determined at the fusion center for making the final decision.

Moreover, most of the multi-dimensional SS techniques proposed in the literature do not consider the effect of channel correlation. Some contributions in the literature have analyzed the performance of the ED technique with correlated multiple antennas. In [33], the authors analyzed the sensing performance of an energy detector when multiple antennas are correlated and it was verified that the sensing performance of the energy detector is degraded when the channels are spatially correlated and the performance degradation is proportional to the level of correlation. In [34], the detection performance of an ED based SS in a CR with multi-antenna correlated channels has been investigated in Nakagami-m fading channel.

In [35], a weighted ED technique and a correlated GLRT detector have been proposed for SS with multi-antenna correlated channels. In the context of eigenvalue-based SS, the effect of spatial correlation in the performance of predicted eigenvalue threshold based SS is analyzed in [13] and it is shown that the detection performance improves in the presence of spatial correlation at the multi-antenna secondary user. However, the theoretical analysis of the effect of channel correlation in the performance of the proposed technique is not presented in [13]. In [36], a new decision threshold for the purpose of SS has been proposed in the presence of noise correlation and in [22] the a.e.p.d.f. of the received signal's covariance matrix has been derived under signal plus noise case. Furthermore, in [37], asymptotic analysis of eigenvaluebased blind SS techniques such as Scaled Largest Eigenvalue (SLE), signal condition number, John's detection, spherical test based detection has been carried out and the performance of these techniques has been evaluated in the presence of noise correlation numerically. Moreover, the contribution in [15] uses the RMT in order to estimate the transmit power of multiple signal sources blindly in multiantenna fading channels.

\section{Signal Model}

We consider a single PU for simplicity of analysis. Let $N$ be the number of samples analyzed by the cognitive user for 
the decision process and $\tau$ the sensing duration. Let $M$ be the number of receive dimensions in the cognitive receiver. From a system-model point of view, this factor can be considered to be the number of antennas in a multiantenna based CR receiver, number of cooperative users with single antenna $\mathrm{RF}$ chain in a cooperative sensing $\mathrm{CR}$ system ${ }^{3}$ and the number of oversampled branches in an oversampling based sensing model as considered in [5,26]. The $M \times N$ received signal matrix $\mathbf{Y}$ in the considered multi-dimensional framework can be written as:

$$
\mathbf{Y}=\left[\begin{array}{c}
\mathbf{y}_{1} \\
\mathbf{y}_{2} \\
\vdots \\
\mathbf{y}_{M}
\end{array}\right]=\left[\begin{array}{cccc}
y_{1}(1) & y_{1}(2) & \ldots & y_{1}(N) \\
y_{2}(1) & y_{2}(2) & \ldots & y_{2}(N) \\
\vdots & \vdots & \ddots & \vdots \\
y_{M}(1) & y_{M}(2) & \ldots & y_{M}(N)
\end{array}\right]
$$

The signal model presented in this paper is generic and can be applicable to all the considered multi-dimensional scenarios.

The PU transmitted signal may suffer from slow or fast fading depending on the nature of wireless fading channel. Furthermore, the channel effect on the transmitted symbol may remain constant or vary depending on the transmit data rate and the sampling rate applied at the CR receiver. Moreover, a constant symbol or random symbols may appear in the receiver depending on the relation between sensing duration $\tau$ and transmitted symbol duration $T_{s}$. Depending on the appearance of constant or random symbol and the nature of wireless channels, the following cases can be considered for modeling the received PU signal at a cognitive terminal. Case 1: $\tau \leq T_{s}$ and channel coefficients vary across receive (i.e., spatial) dimensions but remain constant across temporal dimension: $\mathbf{Y}=\sqrt{p} \hat{\mathbf{h}} \cdot s \cdot \mathbf{1}+\hat{\mathbf{Z}}$, where $\hat{\mathbf{h}}$ is an $M \times 1$ correlated channel vector i.e., $\hat{\mathbf{h}}=[\hat{h}(1), \hat{h}(2), \ldots, \hat{h}(M)]^{T}$, $\hat{\mathbf{Z}} \triangleq\left[\hat{\mathbf{z}}_{1}^{T}, \hat{\mathbf{z}}_{2}^{T}, \ldots, \hat{\mathbf{z}}_{M}^{T}\right]^{T}$ is $M \times N$ correlated noise with $\hat{\mathbf{z}}_{m} \triangleq\left[\begin{array}{llll}\hat{z}_{m}(1) & \hat{z}_{m}(2) & \ldots & \hat{z}_{m}(N)\end{array}\right]$ with $m=1,2, \ldots, M$, $s$ is a constant transmitted symbol, $p$ is the power of transmitted symbol, $\mathbf{1}$ is an $1 \times N$ vector with all elements being 1. Since we assume normalized noise variance, $\mathrm{SNR} \equiv p$.

Case 2: $\tau \leq T_{s}$ and channel coefficients vary across both (spatial and temporal) dimensions: $\mathbf{Y}=\sqrt{p} \hat{\mathbf{H}} s+\hat{\mathbf{Z}}$, where $\hat{\mathbf{H}} \triangleq\left[\hat{\mathbf{h}}_{1}^{T}, \hat{\mathbf{h}}_{2}^{T}, \ldots, \hat{\mathbf{h}}_{M}^{T}\right]^{T}$ with $\hat{\mathbf{h}}_{m} \triangleq\left[\begin{array}{llll}\hat{h}_{m}(1) & \hat{h}_{m}(2) & \ldots & \hat{h}_{m}(N)\end{array}\right]$.

Case 3: $\tau>T_{s}$ and channel coefficients vary across spatial dimension but remain constant across temporal dimension: $\mathbf{Y}=\sqrt{p} \hat{\mathbf{h}} \mathbf{s}+\hat{\mathbf{Z}}$, where $\mathbf{s}$ is an $1 \times N$ PU transmitted signal vector.

Case 4. $\tau>T_{s}$ and channel coefficients vary across both dimensions: $\mathbf{Y}=\sqrt{p} \hat{\mathbf{H}} \mathbf{S}_{d}+\hat{\mathbf{Z}}$, where $\mathbf{S}_{d}$ is an $N \times N$ diagonal transmitted signal matrix with the diagonal $\mathbf{s}=[s(1) \ldots s(N)]$.

We are interested in analyzing cases 2 and 4 in this paper ${ }^{4}$. For our analysis, we assume that transmitted symbols

\footnotetext{
${ }^{3}$ In this context, we consider the PU signal detection phase of the cooperative system assuming perfect reporting channels as in [26].

${ }^{4}$ Cases 1 and 3 involve unit rank Wishart matrices which are straightforward to analyze.
}

are independent and identically distributed (i.i.d.) complex circularly symmetric (c.c.s.) Gaussian symbols. For case 2, the covariance of the transmitted signal can be written as: $\mathbf{R}_{s}=\mathbb{E}\left[s^{2}\right]=1$. Similarly, for case $4, \mathbf{R}_{\mathbf{S}_{d}}=\mathbb{E}\left[\mathbf{S}_{d} \mathbf{S}_{d}^{H}\right]=\mathbf{I}$ assuming that for each sample we get an i.i.d. c.c.s. symbol with $\mathbb{E}\left[s^{2}\right]=1$. We denote the hypotheses of the presence and absence of the PU signal by $\mathbb{H}_{1}$ and $\mathbb{H}_{0}$ respectively. A binary hypothesis testing problem for multi-dimensional sensing techniques can be formulated as:

$$
\begin{aligned}
\mathbb{H}_{0}: \mathbf{Y} & =\hat{\mathbf{Z}} \\
\mathbb{H}_{1}: \mathbf{Y} & =\sqrt{p} \hat{\mathbf{H}} s+\hat{\mathbf{Z}} \quad \text { Case } 2 \\
& =\sqrt{p} \hat{\mathbf{H}} \mathbf{S}_{d}+\hat{\mathbf{Z}} \quad \text { Case } 4
\end{aligned}
$$

Assuming that the source signal is independent from the noise, the covariance matrix of received signal $\mathbf{R}_{\mathbf{Y}}$ can be calculated as [5]:

$$
\begin{aligned}
\mathbf{R}_{\mathbf{Y}} & =\mathbb{E}\left[\mathbf{Y} \mathbf{Y}^{H}\right]=\mathbb{E}\left[(\sqrt{p} \hat{\mathbf{H}} \mathbf{S})(\sqrt{p} \hat{\mathbf{H}} \mathbf{S})^{H}\right]+\mathbb{E}[\hat{\mathbf{Z}} \hat{\mathbf{Z}}]^{H} \\
& =p \mathbb{E}\left[\hat{\mathbf{H}} \hat{\mathbf{H}}^{H}\right]+\mathbf{R}_{\hat{\mathbf{Z}}}
\end{aligned}
$$

where $\mathbf{R}_{\hat{\mathbf{Z}}}=\mathbb{E}\left[\hat{\mathbf{Z}} \hat{\mathbf{Z}}^{H}\right]$. Let us define the sample covariance matrices of the received signal and noise as: $\hat{\mathbf{R}}_{\mathbf{Y}}(N)=$ $\frac{1}{N} \mathbf{Y} \mathbf{Y}^{H}$ and $\hat{\mathbf{R}}_{\hat{\mathbf{Z}}}(N)=\frac{1}{N} \hat{\mathbf{Z}} \hat{\mathbf{Z}}^{H}$.

\section{A. Causes of Correlation}

In practical implementation of a $\mathrm{CR}$, noise and channel may be correlated due to various reasons. Depending on the considered multi-dimensional scenario, the causes of channel and noise correlation may be different. Table I summarizes the types and causes of noise and channel correlation.

1) Causes of Channel correlation: The channel correlation in MIMO/Single Input Multiple Output (SIMO) systems depends on the following two components.

a) Spatial Correlation: In wireless multipath environments, the channels are not always independent from each other but can be correlated due to poor scattering in the propagation path. This type of correlation can be referred as spatial correlation. In such environments, multipath signals tend to leave the transmit antenna array in a range of angular directions and tend to arrive at the receive antenna array from a range of angular directions rather than a single angular direction. The rich scattering in the propagation path decreases the spatial correlation by spreading the signal such that multipath components are received from many different spatial directions [11]. The smaller spacing between antennas in the transmit and receive sides increases the spatial correlation since adjacent antennas receive similar signal components.

b) Antenna Mutual Coupling: Channel correlation also arises due to mutual coupling between the transmit and/or receive antenna elements $[12,38]$. In the transmitter antenna array, antenna mutual coupling causes the input signals to be coupled with the neighbouring antennas. Similarly, the channel correlation may arise due to antenna mutual coupling effect in the receiving antenna arrays. 
TABLE I: Causes of channel/noise correlation in multi-dimensional scenarios

\begin{tabular}{|c|c|c|c|c|}
\hline \multirow[t]{2}{*}{ Cases } & \multicolumn{2}{|c|}{ Channel Correlation } & \multicolumn{2}{|c|}{ Noise Correlation } \\
\hline & Type & Cause & Type & Cause \\
\hline \multirow{2}{*}{$\begin{array}{l}\text { Multi-antenna } \\
\text { SS }\end{array}$} & Spatial Correlation & $\begin{array}{l}\text { Multipath } \\
\text { propagation }\end{array}$ & \multirow[t]{2}{*}{ Filtering } & \multirow{2}{*}{$\begin{array}{l}\text { Autocorrelation } \\
\text { function of the } \\
\text { filter }\end{array}$} \\
\hline & $\begin{array}{l}\text { Antenna mutual } \\
\text { coupling }\end{array}$ & $\begin{array}{l}\text { Transmit/receive } \\
\text { Antenna } \\
\text { separation }\end{array}$ & & \\
\hline \multirow[t]{2}{*}{$\begin{array}{l}\text { Oversampled } \\
\text { SS }\end{array}$} & Oversampling & $\begin{array}{l}\text { Oversampling } \\
\text { operation }\end{array}$ & Filtering & $\begin{array}{l}\text { Autocorrelation } \\
\text { function of the } \\
\text { filter }\end{array}$ \\
\hline & Filtering & $\begin{array}{l}\text { Autocorrelation } \\
\text { function of filter }\end{array}$ & Oversampling & $\begin{array}{l}\text { Oversampling } \\
\text { operation }\end{array}$ \\
\hline Cooperative SS & Spatial correlation & $\begin{array}{l}\text { Collocated nodes in } \\
\text { CR network }\end{array}$ & Filtering & $\begin{array}{l}\text { Autocorrelation } \\
\text { function of the } \\
\text { filter }\end{array}$ \\
\hline
\end{tabular}

2) Causes of Noise correlation: The noise correlation in the receiver may arise due to the following reasons.

a) Filtering: In practice, the received signal is passed through a pulse shaping filter before further processing in the $\mathrm{CR}$ receiver. In this case, the noise which is added to the signal before the filter is also filtered out and it becomes affected by the autocorrelation function of the filter. For example, when a white noise input process with power spectral density $N_{0} / 2$ is the input to a RC filter with time constant RC, the noise is affected by the autocorrelation function of the $\mathrm{RC}$ filter and it becomes colored. The RC filter transforms the input autocorrelation function of white noise into the output autocorrelation function given by [39]; $R_{y}(\nu)=\frac{N_{0}}{4 R C} e^{-\frac{|\nu|}{R C}}$. b) Oversampling: Let the pulse shaping filter has a bandwidth of $W \mathrm{~Hz}$, which is usually equal to the bandwidth of the signal. If we sample at a rate higher than the Nyquist rate i.e., $2 W \mathrm{~Hz}$, the noise process in the output becomes correlated even if the input noise process is white.

3) Causes of both channel/noise correlation: The filtering and oversampling operations may also cause channel correlation in addition to noise correlation since the received signal passes through the same filter and the same oversampling operation. If we want to include the effect of filtering and oversampling operations in the performance of the SS and SNR estimation techniques, both channel and noise correlation need to be considered in the analysis.

\section{B. Noise Correlation Modeling}

To analyze the noise correlation, we consider noise correlation across the receive dimensions and not across the temporal dimension. To model this scenario, we consider the one-sided noise correlation model as in [22]. We model the correlated noise as: $\hat{\mathbf{Z}}=\boldsymbol{\Theta}^{1 / 2} \mathbf{Z}$, where $\mathbf{Z}$ is an $M \times N$ matrix with c.c.s. i.i.d. Gaussian entries with zero mean and unit variance, representing the white noise and $\Theta^{1 / 2} \Theta^{1 / 2}=\boldsymbol{\Theta}=\mathbb{E}\left[\hat{\mathbf{Z}} \hat{\mathbf{Z}}^{H}\right]$. It can be noted that since $\mathbf{Z} \sim \mathcal{C N}(0, \mathbf{I}), \mathbf{Z Z}^{H}$ follows an uncorrelated Wishart distribution i.e., $\mathbf{Z Z}^{H} \sim \mathcal{W}_{M}\left(\mathbf{R}_{\mathbf{Z}}, N\right)$. To ensure that $\Theta$ does not affect the noise power, the normalization $(1 / M)$ trace $\{\boldsymbol{\Theta}\}=1$ is considered.

Since the output autocorrelation function of the RC filter resembles an exponential function, we define the components of $\Theta$ using an exponential covariance model, which is given by [23];

$$
\theta_{i j} \sim \begin{cases}\varsigma^{(j-i)}, & i \leq j \\ \left(\varsigma^{(i-j)}\right)^{*}, & i>j\end{cases}
$$

where $\theta_{i j}$ is the $(i, j)$ th element of $\Theta$ and $\varsigma \in \mathbb{C}$ is the correlation coefficient with $|\varsigma| \leq 1$.

\section{Channel Correlation Modeling}

As in [24], we use the one-sided correlation model to model channel correlation. In this paper, we focus on the correlation across channel receive dimensions and not on the temporal correlation $^{5}$. We model the channel as: $\hat{\mathbf{H}}=\boldsymbol{\Phi}^{1 / 2} \mathbf{H}$, where $\mathbf{H}$ is an $M \times N$ matrix with c.c.s. i.i.d. Gaussian entries with zero mean and unit variance and $\boldsymbol{\Phi}^{1 / 2} \boldsymbol{\Phi}^{1 / 2}=\boldsymbol{\Phi}=\mathbb{E}\left[\hat{\mathbf{H}} \hat{\mathbf{H}}^{H}\right]$. It can be noted that since $\mathbf{H} \sim \mathcal{C N}(0, \mathbf{I}), \mathbf{H H}^{H}$ follows uncorrelated Wishart distribution i.e., $\mathbf{H H}^{H} \sim \mathcal{W}_{M}\left(\mathbf{R}_{\mathbf{H}}, N\right)$. To ensure that $\boldsymbol{\Phi}$ does not affect the channel power, we consider the following normalization: $(1 / M) \operatorname{trace}\{\boldsymbol{\Phi}\}=1$, where each component of $\boldsymbol{\Phi}$ is modeled with the exponential covariance model given by (4).

\section{EigenVAlue-BASED SS TEChNiques}

In [5], the TW distribution is used as a statistical model for the largest eigenvalue and a combination of the TW based and the MP-based approaches is used to find the approximate distribution of random SCN. Subsequently, this distribution

\footnotetext{
${ }^{5}$ The analysis presented in this paper can be straightforwardly extended to the time correlated noise/channel case assuming the exponential correlation model still holds. In this case, the one-sided correlation model can be applied on the right hand side of the noise/channel matrix instead of left hand side.
} 
is used to derive the relationship between an expression for $P_{f}$ and the threshold. The difference between MP-based approach and TW approach is that MP-based approach uses deterministic asymptotic bounds for the a.e.p.d.f. support while the TW approach uses the pdf of the maximum/minimum eigenvalue for finite dimensions. In the following subsections, we briefly review the asymptotic MP-based approach [25], semi-asymptotic MME and EME approaches [5] for their use in our context.

\section{A. Maximum to Minimum Eigenvalue (MME)}

The decision statistic for the MME method is the ratio of the maximum eigenvalue to the minimum eigenvalue i.e., SCN. Based on the distribution used for analyzing the bounds for the maximum and the minimum eigenvalues, this technique can be categorized into the following sub-techniques.

1) Asymptotic $M M E$ : In this approach, both the maximum and the minimium eigenvalues are calculated based on the asymptotical properties of Wishart matrices. The minimum and the maximum eigenvalues of $\hat{\mathbf{R}}_{\mathbf{Y}}(N)$ asymptotically converge almost surely to $a=(1-\sqrt{\beta})^{2}$ and $b=(1+\sqrt{\beta})^{2}$ respectively, in the limit $M, N \rightarrow \infty$ with ratio index $\beta=$ $N / M$ [8]. The parameters $a$ and $b$ can be regarded as the bounds of the MP distribution and based on these MP bounds, the absence or presence of a PU signal can be decided under the white noise scenario [25]. Under white noise scenarios, the decision statistic can be calculated using the MP law as [22]:

$$
\text { decision }= \begin{cases}\mathbb{H}_{0}, & \text { if } \frac{\lambda_{\max }\left(\hat{\mathbf{R}}_{\mathbf{Y}}(\mathrm{N})\right)}{\lambda_{\min }\left(\hat{\mathbf{R}}_{\mathbf{Y}}(\mathrm{N})\right)} \leq \frac{b}{a} \\ \mathbb{H}_{1}, & \text { otherwise }\end{cases}
$$

where $\lambda_{\max }\left(\hat{\mathbf{R}}_{\mathbf{Y}}(N)\right)$ and $\lambda_{\min }\left(\hat{\mathbf{R}}_{\mathbf{Y}}(N)\right)$ denote the maximum and minimum eigenvalues of $\hat{\mathbf{R}}_{\mathbf{Y}}(N)$ respectively. This asymptotic approach does not have Constant False Alarm Rate (CFAR) property since the threshold is not a function of the false alarm rate.

2) Semi-asymptotic MME: This MME approach is semiasymptotic in nature since the bound for the maximum eigenvalue is calculated based on the TW distribution instead of the asymptotic distribution while the minimum eigenvalue is evaluated based on asymptotic analysis [26]. The binary hypothesis testing problem for this technique can be written as:

$$
\text { decision }= \begin{cases}\mathbb{H}_{0}, & \text { if } \frac{\lambda_{\max }\left(\hat{\mathbf{R}}_{\mathbf{Y}}(\mathrm{N})\right)}{\lambda_{\min }\left(\hat{\mathbf{R}}_{\mathbf{Y}}(\mathrm{N})\right)} \leq \gamma_{1} \\ \mathbb{H}_{1}, & \text { otherwise }\end{cases}
$$

where $\gamma_{1}$ is the decision threshold for MME method, which can be written as [26]:

$$
\gamma_{1}=\frac{b}{a} \cdot\left(1+\frac{(\sqrt{N}+\sqrt{M})^{-2 / 3}}{N M^{1 / 6}} F_{T W 2}^{-1}\left(1-P_{f}\right)\right),
$$

where $F_{T W 2}^{-1}$ is the inverse Tracy-Widom CDF of order 2.

\section{B. Energy with Minimum Eigenvalue (EME)}

The average power of the received signal is nearly same as the average eigenvalue $\left(\lambda_{\text {avg }}\right)$ of the received signal's covariance matrix [5]. The binary hypothesis testing problem based on EME technique can be written as:

$$
\text { decision }= \begin{cases}\mathbb{H}_{0}, & \text { if } \frac{\lambda_{\text {avg }}\left(\hat{\mathbf{R}}_{\mathbf{Y}}(N)\right)}{\lambda_{\min }\left(\hat{\mathbf{R}}_{\mathbf{Y}}(N)\right)} \leq \gamma_{2} \\ \mathbb{H}_{1}, & \text { otherwise }\end{cases}
$$

where $\gamma_{2}$ is the decision threshold for the EME method, which is given by [5];

$$
\gamma_{2}=\left(\sqrt{\frac{2}{M N}} Q^{-1}\left(P_{f}\right)+1\right) \frac{N}{(\sqrt{N}-\sqrt{M})^{2}},
$$

where $Q^{-1}$ represents the inverse Q-function with $Q(t)=$ $\frac{1}{\sqrt{2 \pi}} \int_{t}^{\infty} e^{-u^{2} / 2} d u$.

Remark 4.1: It can be noted that GLRT like algorithms such as SLE (i.e., maximum eigenvalue/average eigenvalue) have been investigated in $[40,41]$. Furthermore, the effect of noise correlation on different eigenvalue-based blind techniques including the SLE detector has been analyzed in [37], where it is shown that the SLE technique performs better than other eigenvalue-based techniques for a variety of scenarios and even in the presence of noise correlation. Therefore, we simply provide an overview of MME/EME techniques and evaluate their performances in the presence of channel/noise correlation numerically in Section VII.

\section{Channel Correlation Analysis using RMT}

For the purpose of completeness, we state the following RMT theorem which is going to be used in our analysis.

Theorem 5.1: [24] Let $\Theta$ be a positive definite matrix which is normalized as: $(1 / M) \operatorname{trace}\{\boldsymbol{\Theta}\}=1$, and whose asymptotic spectrum has the p.d.f.

$$
f_{\Theta}(\lambda)=\frac{1}{2 \pi \mu \lambda^{2}} \sqrt{\left(\frac{\lambda}{\sigma_{1}}-1\right)\left(1-\frac{\lambda}{\sigma_{2}}\right)}
$$

with $\sigma_{1} \leq \lambda \leq \sigma_{2}$ and $\mu=\frac{\left(\sqrt{\sigma_{2}}-\sqrt{\sigma_{1}}\right)^{2}}{4 \sigma_{1} \sigma_{2}}$. If $\mathbf{F}$ is an $M \times N$ standard complex Gaussian matrix and $\frac{1}{N} \mathbf{F F}^{H}$ follows the MP law [8], then as $M, N \rightarrow \infty$ with $\frac{N}{M} \rightarrow \beta$, the asymptotic eigenvalue distribution of $\mathbf{W}=\Theta^{1 / 2} \mathbf{F} \mathbf{F}^{H} \Theta^{1 / 2}$ has the following p.d.f.

$$
f_{\mathbf{W}}(\lambda)=(1-\beta)^{+} \delta(\lambda)+\frac{\sqrt{(\lambda-\tilde{a})^{+}(\tilde{b}-\lambda)^{+}}}{2 \pi \lambda(1+\lambda \mu)}
$$

where $\tilde{a}=1+\beta+2 \mu \beta-2 \sqrt{\beta} \sqrt{(1+\mu)(1+\mu \beta)}, \tilde{b}=$ $1+\beta+2 \mu \beta+2 \sqrt{\beta} \sqrt{(1+\mu)(1+\mu \beta)}, \delta($.$) is a Dirac delta$ function and $(1-\beta)^{+} \delta(\lambda)$ represents the cardinality of zero eigenvalues which can occur if $M>N$. The parameters $\tilde{a}$ and $\tilde{b}$ correspond to $\lambda_{\min }^{\infty}$ and $\lambda_{\max }^{\infty}$ respectively and the ratio $\tilde{b} / \tilde{a}$ defines the SCN of $\mathbf{W}$.

Proof: For detailed proof, see [24].

The above theorem is applicable for noise covariance matrix $\Theta$ in case of noise correlation and for channel covariance matrix $\boldsymbol{\Phi}$ in case of channel correlation. The eigenvalue spread of $\Theta$ or $\Phi$ is related to the degree of noise or channel covariance i.e., a zero eigenvalue spread corresponds to a zero-covariance model and higher spreads are associated with higher covariance models. In (11), the parameter $\mu$ controls the 
degree of covariance and varies the support of the distribution i.e., for $\mu=0, \tilde{a}=a$ and $\tilde{b}=b$, where $a=(1-\sqrt{\beta})^{2}$ and $b=(1+\sqrt{\beta})^{2}$ are the supports of the MP distribution [8]. For the exponential covariance model as stated in [24], the parameter $\mu$ is related to the correlation coefficient $\varsigma$ with the following relation: $\mu=\frac{\varsigma^{2}}{1-\varsigma^{2}}$. Furthermore, the SCN is related to $\varsigma$ with the relation $\mathrm{SCN}=\frac{1+\varsigma}{1-\varsigma}$. To calculate $\mu$ in a practical cognitive receiver, the value of $\varsigma$ for noise correlation case can be determined based on some empirical model constructed from measurements such as [22] and its value for channel correlation case can be determined using channel estimation techniques like [42]. The received signal matrix under correlated channel and white noise case can be written as:

$$
\mathbf{Y}=\sqrt{p} \boldsymbol{\Phi}^{\frac{1}{2}} \mathbf{H} \mathbf{S}_{d}+\mathbf{Z}
$$

Assuming that signal and noise are independent, for very large value of $N$, the sample covariance matrix of received signal under considered scenario in the presence of channel correlation can be approximated as [5]:

$$
\lim _{N \rightarrow \infty} \hat{\mathbf{R}}_{\mathbf{Y}}(N) \approx p \boldsymbol{\Phi}^{\frac{1}{2}} \mathbf{H} \mathbf{H}^{H} \boldsymbol{\Phi}^{\frac{1}{2}}+\hat{\mathbf{R}}_{\mathbf{Z}}
$$

\section{A. Methodology}

The a.e.p.d.f. of the received signal's covariance matrix under the $\mathbb{H}_{1}$ hypothesis can be used to estimate the PU SNR by a CR. To calculate the a.e.p.d.f. of $\hat{\mathbf{R}}_{\mathbf{Y}}(N)$, we need to know the Stieltjes transform of its asymptotic density function. In this subsection, we provide a generalized methodology to derive the a.e.p.d.f. for $\hat{\mathbf{R}}_{\mathbf{Y}}(N)$ in the presence of channel correlation referring to the case considered in (13).

Due to noncommutative nature of random matrices, it's not straightforward to calculate the eigenvalue distributions of the received signal $\mathbf{Y}$ by knowing the eigenvalue distribution of covariance matrices of $\boldsymbol{\Phi}, \mathbf{H}$ and $\mathbf{Z}$ in (13). Using free probability analysis, the asymptotic spectrum of the sum or product can be obtained from the individual asymptotic spectra without involving the structure of the eigenvectors of the matrices [8] under a asymptotic freeness condition. The asymptotic eigenvalue distribution of $\mathbf{Y}^{6}$ in our context can be obtained by applying $\Sigma$ transform and R transform [8]. In (13), since $\boldsymbol{\Phi}$ is a deterministic matrix and $\mathbf{H} \mathbf{H}^{H}$ is a Wishart random matrix, they are asymptotically free (see Example 2.34, [8]). As a result, the combined a.e.p.d.f. of the term $p \boldsymbol{\Phi}^{\frac{1}{2}} \mathbf{H} \mathbf{H}^{H} \boldsymbol{\Phi}^{\frac{1}{2}}$ in (13) can be obtained by applying multiplicative free convolution property of $\Sigma$ transform in the following way [44].

$$
\Sigma_{p \hat{\mathbf{R}}_{\hat{\mathbf{H}}}}(z)=\Sigma_{\boldsymbol{\Phi}}(z) \cdot \Sigma_{p \hat{\mathbf{R}}_{\mathbf{H}}}(z),
$$

where $\Sigma_{\boldsymbol{\Phi}}$ and $\Sigma_{p \hat{\mathbf{R}}_{\mathbf{H}}}$ are the $\Sigma$ transforms of the densities of eigenvalues of $\boldsymbol{\Phi}$ and $p \hat{\mathbf{R}}_{\mathbf{H}}$ respectively. Since $\boldsymbol{\Phi}$ is a square matrix, $\boldsymbol{\Phi}^{1 / 2} \mathbf{H} \mathbf{H}^{H} \boldsymbol{\Phi}^{1 / 2}$ and $\boldsymbol{\Phi} \mathbf{H} \mathbf{H}^{H}$ have identical eigenvalues [8]. Furthermore, $\mathbf{\Phi} \mathbf{H} \mathbf{H}^{H}$ and $\mathbf{Z} \mathbf{Z}^{H}$ are independent and

\footnotetext{
${ }^{6}$ The analysis carried out in this paper is based on the assumption that both dimensions $M$ and $N$ go to infinity with some finite ratio $\beta=N / M$. However, as noted in [25] and [43], the asymptotic analysis provide valid approximations even for finite dimensions while providing more tractable solutions.
}

$\mathbf{Z} \mathbf{Z}^{H}$ is a Wishart matrix. As a result, $\mathbf{\Phi} \mathbf{H} \mathbf{H}^{H}$ and $\mathbf{Z} \mathbf{Z}^{H}$ are asymptotically free due to unitarily invariance [9]. Then the combined $\mathrm{R}$ transform of $\hat{\mathbf{R}}_{\mathrm{Y}}$ can be found from the $\mathrm{R}$ transforms of $\hat{\mathbf{R}}_{\mathbf{Z}}$ and $p \hat{\mathbf{R}}_{\hat{\mathbf{H}}}$ using additive free convolution property of $\mathrm{R}$ transform in the following way [45].

$$
\mathcal{R}_{\hat{\mathbf{R}}_{\mathbf{Y}}}(z)=\mathcal{R}_{\hat{\mathbf{R}}_{\mathbf{Z}}}(z)+\mathcal{R}_{p \hat{\mathbf{R}}_{\hat{\mathbf{H}}}}(z) .
$$

Since $\hat{\mathbf{R}}_{\mathbf{Z}}$ follows MP law, its $\mathrm{R}$ transform can be written as the $\mathrm{R}$ transform of the MP law and is given by (24). Using the transformations as described in the preliminaries, we get the Stieltjes transform of $\hat{\mathbf{R}}_{\mathbf{Y}}$ (See Appendix B for detailed procedure). The Stieltjes transforms obtained for different correlated scenarios are provided in Section VI. The a.e.p.d.f. of $\hat{\mathbf{R}}_{\mathbf{Y}}$ is then obtained by determining the imaginary part of the Stieltjes transform $\mathcal{S}_{\hat{\mathbf{R}}_{\mathbf{Y}}}$ for real arguments in the following way.

$$
f(x)=\lim _{y \rightarrow 0^{+}} \frac{1}{\pi} \operatorname{Im}\left\{\mathcal{S}_{\hat{\mathbf{R}}_{\mathbf{Y}}}(x+j y)\right\} .
$$

\section{Vi. Main Analytical Results}

In this section, firstly, we mention two theorems (Theorems 6.1 and 6.2) for the white and correlated noise cases from [22]. These results will be used in our numerical analysis in order to compare the performance of the considered cases in this paper. Then we present the main results of our analysis (Theorems 6.3 and 6.4). The proofs of these theorems are postponed to the Appendix to improve the continuity of this manuscript.

Theorem 6.1: The Stieltjes transform $\mathcal{S}_{\hat{\mathbf{R}}_{\mathbf{Y}}}$ of the asymptotic distribution of eigenvalues of $\frac{1}{N} \mathbf{Y} \mathbf{Y}^{H}$ where $\mathbf{Y}=$ $\mathbf{H S}_{d}+\mathbf{Z}$ can be obtained for any $z \in \mathbb{C}$ by solving a cubic polymonial having the following coefficients

$$
\begin{aligned}
& c_{0}=1, \\
& c_{1}=(1-\beta)(1-p)+z, \\
& c_{2}=p(-2 \beta+z+1)+z, \\
& c_{3}=p z,
\end{aligned}
$$

where $c_{n}$ is the $n$th order coefficient of the polymonial, $\beta=\frac{N}{M}$ and $p$ is the SNR of the transmitted PU signal.

Proof: The proof of this theorem can be found in [22].

Theorem 6.2: The Stieltjes transform $\mathcal{S}_{\hat{\mathbf{R}}_{\mathbf{Y}}}$ of the asymptotic distribution of eigenvalues of $\frac{1}{N} \mathbf{Y} \mathbf{Y}^{H}$ where $\mathbf{Y}=$ $\mathbf{H S}_{d}+\hat{\mathbf{Z}}$ can be obtained for any $z \in \mathbb{C}$ by solving a quartic polymonial with the following coefficients

$$
\begin{aligned}
& c_{0}=1+\mu, \\
& c_{1}=2 p(1+\mu(1-\beta))+z(1+2 \mu)-\beta(1+p)+1, \\
& c_{2}=p^{2}\left(\mu(1-\beta)^{2}+1-\beta\right)+2 p(1+z+\mu z(2-\beta))+z-3 p \beta+z^{2} \mu, \\
& c_{3}=2 z \mu p(z-p \beta)+p^{2}(1+2 z \mu+z-2 \beta)+2 z p, \\
& c_{4}=z p^{2}(1+\mu z),
\end{aligned}
$$

where $\mu=\frac{\varsigma^{2}}{1-\varsigma^{2}}$, defines the degree of covariance of the noise covariance matrix $\Theta$.

Proof: The proof of this theorem can be found in [22].

Theorem 6.3: The Stieltjes transform $\mathcal{S}_{\hat{\mathbf{R}}_{\mathbf{Y}}}$ of the asymptotic distribution of eigenvalues of $\frac{1}{N} \mathbf{Y} \mathbf{Y}^{H}$ where $\mathbf{Y}=$ 
$\hat{\mathbf{H}} \mathbf{S}_{d}+\mathbf{Z}$ can be obtained for any $z \in \mathbb{C}$ by solving a quartic polymonial with the following coefficients

$c_{0}=1+\mu$,

$c_{1}=2 \mu(z+1-\beta)-\beta(p+1)+p+z+2$,

$c_{2}=p(z-3 \beta+2)+\beta(\mu(\beta-2 z-2)-1)+\mu(z(z+\mu)+1)+2 z$,

$c_{3}=-2 p(\beta-z)+2 z \mu(1-\beta+z)+p+z$,

$c_{4}=z(p+\mu z)$,

where the parameter $\mu$ defines the degree of covariance of the channel covariance matrix $\boldsymbol{\Phi}$.

Proof: See Appendix B.

Theorem 6.4: The Stieltjes transform $\mathcal{S}_{\hat{\mathbf{R}}_{\mathbf{Y}}}$ of the asymptotic distribution of eigenvalues of $\frac{1}{N} \mathbf{Y} \mathbf{Y}^{H}$ where $\mathbf{Y}=$ $\hat{\mathbf{H}} \mathbf{S}_{d}+\hat{\mathbf{Z}}$ can be obtained for any $z \in \mathbb{C}$ by solving a quartic polymonial with the following coefficients

$c_{0}=\mu(\mu+2)(\mu+1)^{2}$,

$c_{1}=-\mu(\mu+1)\left(\left(-4 \mu^{2}-8 \mu-2\right) z+(1+p)((2 \beta-2) \mu-3+2 \beta)\right)$,

$c_{2}=\mu\left(6 z^{2} \mu^{3}-2 z((2 p+2) \beta-6 z-3(p+1)) \mu^{2}+\mu\left(5 z^{2}\right.\right.$

$\left.+(-2(1+p)(-5+2 \beta)) z+(p-1)^{2} \beta^{2}+(p+1)^{2}(1-2 \beta)+p\right)$

$\left.+p^{2}(1-\beta)+(3 z-6 \beta+4) p+3 z+1-\beta\right)$,

$c_{3}=\mu p^{2}(1+z(1+2 \mu)-2 \beta)+\mu^{2} \beta\left(-2 z(1+p)^{2}-2 z^{2} \mu(1+p)\right)$

$+\mu z(1+4 z \mu(1+3 p))+2+\mu^{2} z^{2}\left((1+p)(5+6 \mu)+4 \mu^{2} z\right)$,

$c_{4}=z^{2} \mu^{2}\left((1+p)^{2}+p+2 z \mu\right)+z \mu p\left(p+2 z^{2} \mu^{2}+1\right)+z^{4} \mu^{4}$,

where the parameter $\mu$ defines the degree of covariance of the noise covariance matrix $\Theta$ and the channel covariance matrix $\Phi$.

Proof: See Appendix C.

Remark 6.1: The polymonial for calculating the Stieltjes transform considering different values of $\mu$ for noise and channel covariance matrices can be obtained using similar procedure. We do not include this polymonial in this paper due to lack of space.

Remark 6.2: We can find the roots of the above polymonials (18), (19) and (20) in closed forms. The closed form is not specifically written in this paper because it includes many terms which provide no further insight. In practice, we can just solve these polymonials with a mathematical software for finding the Stieltjes transforms under considered scenarios.

\section{A. SNR Estimation Method}

Based on the analysis presented in the above section, firstly, we present the SNR estimation method for estimating the PU SNR in the presence of channel correlation and in the presence of both channel/noise correlation. Then for evaluating the SNR estimation performance, we consider the following four cases: (i) uncorrelated channel plus white noise, (ii) uncorrelated channel plus correlated noise, (iii) correlated channel plus white noise, and (iv) correlated channel plus correlated noise. For comparison purpose, we consider both uncorrelated (Case I) and noise correlated (Case II) cases from [22].

Based on the polymonials of the Stieltjes transforms specified in the above section, the supports for the corresponding a.e.p.d.f. are obtained using (16) ${ }^{7}$. The support for the a.e.p.d.f. of $\hat{\mathbf{R}}_{\mathbf{Y}}$ under uncorrelated channel plus correlated noise case

\footnotetext{
${ }^{7}$ We select the imaginary root which complies with the definition and properties of Stieltjes transform (See Appendix A).
}

is calculated based on (18). Similarly, for correlated channel plus white noise case is obtained using polymonial (19) and for both correlated case is obtained using polymonial (20). It can be noted that the value of $\varsigma$ for correlated channel can be found with channel estimation methods such as [42] and its value for correlated noise can be found by carrying out measurements in the sensing module like [22]. Since we know the value of $\beta$ and we can measure the value of $\varsigma$, we can estimate the value of $p$ by sensing the maximum eigenvalue of $\hat{\mathbf{R}}_{\mathbf{Y}}$. Furthermore, it can be noted that the parameters $M, N$ and $\varsigma$ are assumed as operating parameters of the CR sensing module.

For convenience, a lookup table (Table II) is provided in order to estimate the PU SNR (see Section VII). In the lookup table, we present the maximum eigenvalues of received signal's covariance matrix for the above four cases for different values of SNR and $\beta$. With the help of this table, we can estimate PU SNR based on the maximum eigenvalue of received signal's covariance matrix. Based on this estimated SNR, we could potentially design suitable underlay transmission strategy for secondary transmission as described in Section I. In Section VII, we provide the normalized MSE versus SNR plot to evaluate the performance of this estimation technique. To clarify the above process, we include algorithms for lookup table formation and for PU SNR estimation below.

\section{Algorithm for lookup table formation}

1) Select $N, M$ and calculate $\beta=N / M$.

2) Find $\varsigma$ using channel estimation and noise measurement methods like [42] [22].

3) Calculate $\mu=\frac{\varsigma^{2}}{1-\varsigma^{2}}$.

4) Select $p$ range e.g., $-10 \mathrm{~dB}$ to $5 \mathrm{~dB}$.

5) Evaluate $\mathcal{S}_{\mathbf{R}_{\mathbf{Y}}}$ using (19).

6) Find $\lambda_{\max }\left(\mathbf{R}_{\mathbf{Y}}\right)$ using (16).

7) Store all $\lambda_{\max }\left(\mathbf{R}_{\mathbf{Y}}\right)$ and corresponding $p$ e.g., Table II

Algorithm for SNR estimation

1) Calculate instantaneous $\hat{\mathbf{R}}_{\mathbf{Y}}(N)=\frac{1}{N} \mathbf{Y} \mathbf{Y}^{H}$.

2) Calculate $\lambda_{\max }\left(\hat{\mathbf{R}}_{\mathbf{Y}}\right)$.

3) Find $p$ corresponding to $\lambda_{\max }$ from lookup table.

4) Use suitable interpolation for any intermediate $\lambda_{\max }$.

\section{NUMERICAL RESULTS}

\section{A. Performance Metrics}

In this section, firstly, we study the performance of eigenvalue-based SS techniques in the presence of noise and/or channel correlation. The performance metrics used are (i) probability of correct decision, (ii) probability of correct detection and (iii) probability of a false alarm. These metrics can be defined in the following way. Let $P\left(\mathbb{H}_{i} ; \mathbb{H}_{j}\right)$ indicate the probability of deciding hypothesis $\mathbb{H}_{i}$ when hypothesis $\mathbb{H}_{j}$ is true with $\{i, j\} \in\{0,1\}$. The probability of detection $\left(P_{d}\right)$ can be defined as: $P_{d}=P\left(\mathbb{H}_{1} ; \mathbb{H}_{1}\right)$ and the $P_{f}$ can be defined as: $P_{f}=P\left(\mathbb{H}_{1} ; \mathbb{H}_{0}\right)$ [46]. Then the probability of correct decision is defined as: $\left(P\left(\mathbb{H}_{1} ; \mathbb{H}_{1}\right)+P\left(\mathbb{H}_{0} ; \mathbb{H}_{0}\right)\right) / 2$ i.e., $P_{d}+\left(1-P_{f}\right) / 2$. In other words, it depicts how many correct 
decisions are made out of the total considered realizations under both hypotheses ${ }^{8}$. In the presented simulation results, $10^{3}$ realizations were considered. We consider Rayleigh fading channel in our simulation model and the coefficients of uncorrelated channel $\mathbf{H}$ are generated from random complex numbers whose real and imaginary components are i.i.d. Gaussian variables. Furthermore, the correlated channel $\hat{\mathbf{H}}$ is generated by applying the covariance matrix $\boldsymbol{\Phi}$.

To evaluate the performance of the proposed SNR estimation method in the presence of noise or/and channel correlation, normalized MSE is used and it is defined as:

$$
\mathrm{MSE}=\frac{\mathbb{E}\left[(\hat{p}-p)^{2}\right]}{p^{2}},
$$

where $\hat{p}$ is the estimated SNR with the proposed method and $p$ is the actual SNR.

\section{B. Results}

Figure 1 shows the performance of MME and EME techniques for correlated channel and white noise case in terms of $P_{d}$ versus SNR (SCN $=3$ ). For the comparison of MME and EME techniques in the presence of channel correlation, the fixed false alarm rate of 0.07 was considered and the detection threshold was calculated using eqns. (7) and (9) respectively. Then the value of $P_{d}$ was calculated numerically based on the calculated thresholds for different SNR values in the considered range (from $-15 \mathrm{~dB}$ to $5 \mathrm{~dB}$ ). From the figure, it can be noted that the detection performance improves in the presence of channel correlation as concluded in [13]. It was noted in [22] that the sensing performance of the MME/EME techniques and the MP-based asymptotic technique degrades in the presence of noise correlation. Figure 2 shows the performance of the asymptotic MME technique for different cases with the MP bounds i.e., $[a, b]$ and new bounds proposed in [22] i.e., $[\tilde{a}, \tilde{b}]$ in terms of probability of correct decision. The decision for the MME technique using the MP bounds was calculated based on (5). Similarly, the decision for the MME technique using new bounds was calculated using $\tilde{b} / \tilde{a}$, obtained from the supports of (11), as the decision threshold instead of $b / a$ in (5). The detailed analysis for this decision process can be found in [22]. From the figure, it can be noted that the MP bounds do not perform well in presence of noise correlation and new bounds provide better sensing performance in this scenario. In the presence of spatial correlation, the sensing performance improves with the MP bounds and the performance becomes slightly worse with new bounds. Furthermore, it can be noted that the sensing with new bounds increases sensing performance in the presence of both channel/noise correlation. Similar to the performance results obtained in [13], we note that the performance of the considered eigenvalue-based techniques improve in the presence of spatial correlation. This is due to the reason that the presence of spatial correlation strengthens the eigenvalues of the received signal's matrix under the $\mathbb{H}_{1}$ hypothesis compared to the uncorrelated case. Since the contribution

\footnotetext{
${ }^{8}$ Since threshold is fixed in our scenario and noise correlation affects the value of $P_{f}$, we consider number of correct decisions under both hypotheses.
}

of the signal eigenvalues is improved in comparison to the noise eigenvalues in the presence of spatial correlation, the probability of detection improves in the presence of spatial correlation in eigenvalue-based techniques.

Remark 7.1: As noted in $[33,35]$, the performance of the ED technique degrades in the presence of spatial correlation. The different effects in the performances of the ED and the MME/EME techniques due to the presence of spatial correlation come from the fact that in the ED, noise power is determined completely by the $\mathbb{H}_{0}$ hypothesis while the denominator term in MME/EME techniques (i.e., the minimum eigenvalue) is determined from the received signal's covariance matrix under the $\mathbb{H}_{1}$ hypothesis. More specifically, in the EME/MME techniques, both the numerator and denominator terms vary in the presence of spatial correlation while the noise power does not depend on the spatial correlation for the case of ED.

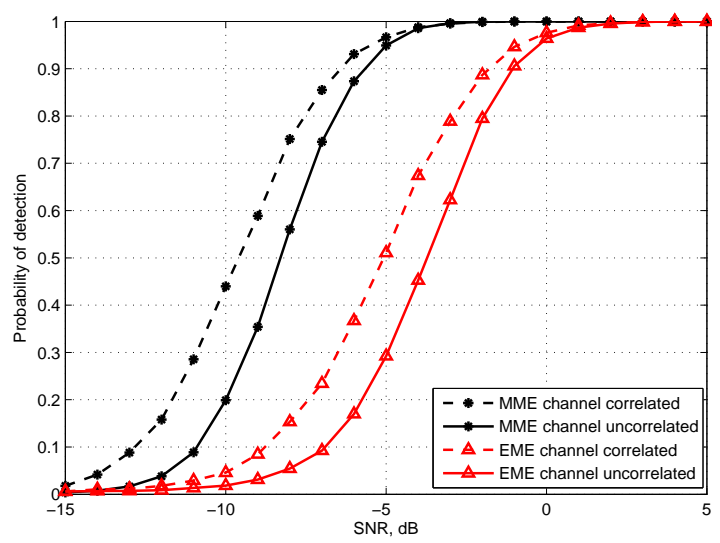

Fig. 1: $P_{d}$ versus SNR (dB) for MME and EME techniques in channel uncorrelated and correlated scenarios $\left(\beta=6, P_{f}=0.07, \mathrm{SCN}=3, N=60\right)$

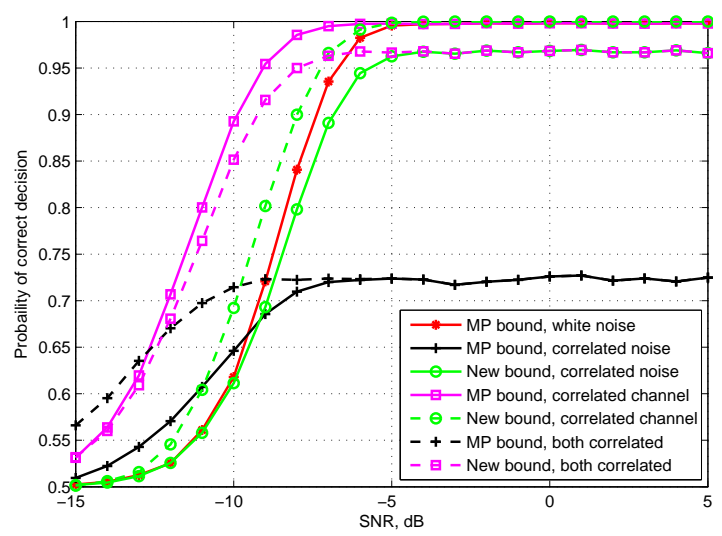

Fig. 2: Probability of correct decision versus SNR for asymptotic MME method $(\beta=6, \mathrm{SCN}=3, N=60)$

Table II shows the lookup table for different values of SCN of the channel/noise covariance matrix and SNRs. For all the considered cases, the maximum eigenvalue corresponding to the particular value of SNR was obtained by solving the polymonial expressions provided in Section VI with the help of a mathematical software. This table can be used to estimate the PU SNR on the values of SCN and $\beta$ for all the considered 


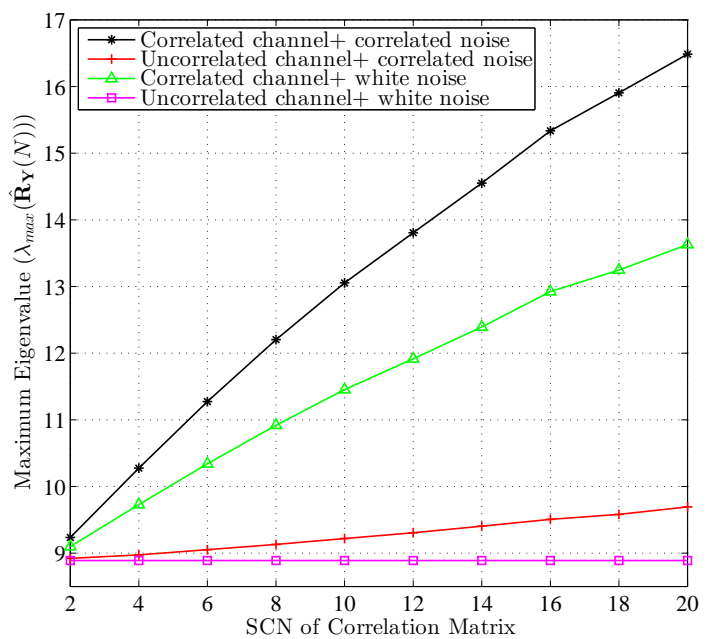

Fig. 3: Maximum Eigenvalue versus SCN of covariance matrix for different cases $(\beta=1, N=100, \mathrm{SNR}=3 d B)$

cases. For example, if the value of $\operatorname{SCN}$ is $2, \beta$ is 1 and the maximum eigenvalue of sample covariance matrix of received signal i.e., $\frac{1}{N} \mathbf{Y} \mathbf{Y}^{H}$ is 4.79 in case II, we can then estimate that PU SNR is $-2 \mathrm{~dB}$ and intermediate values can be calculated through interpolation.

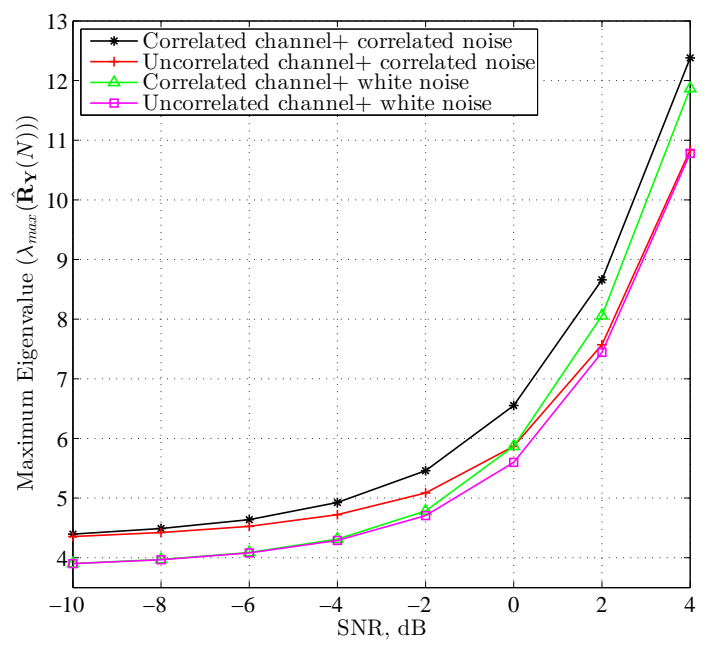

Fig. 4: Maximum Eigenvalue versus SNR for different cases $(\beta=1, N=100, \mathrm{SCN}=4)$

Figure 3 shows the maximum eigenvalue of the received signal's covariance matrix versus $\mathrm{SCN}$ of the covariance matrix for different cases $(\beta=1, N=100, \mathrm{SNR}=3 \mathrm{~dB})$. From the figure, it can be noted that the maximum eigenvalue increases with the SCN for all correlated cases and the rate of increase for both channel/noise correlated case is higher than other two individually correlated cases. Furthermore, the rate of increase of the maximum eigenvalue is higher for the channel correlated case than the noise correlated case at SNR value of $3 \mathrm{~dB}$. Figure 4 shows the maximum eigenvalue versus $\mathrm{SNR}$ for different cases $(\beta=1, \mathrm{SCN}=4, N=100)$. From the figure, it can be noted that the maximum eigenvalue increases with the SNR for all considered cases with the higher rate in the high SNR region. Furthermore, the rate of increase is higher for both correlated case than other cases. From the curves for correlated channel plus white noise case and uncorrelated channel plus correlated noise case, it can be noted that the effect of noise correlation is dominant at lower values of SNR and the channel correlation effect becomes more than that of noise correlation at high SNR values (after $0 \mathrm{~dB}$ in Fig. 4). Furthermore, it can be noted that at the value of $\mathrm{SNR}=0 \mathrm{~dB}$ i.e., $p=1$, effects of channel correlation and noise correlation are equivalent for identical $\varsigma$.

Figure 6 shows the normalized MSE versus SNR with and without the knowledge of noise or/and channel correlation ( $\beta=1, \mathrm{SCN}=4, N=100)$. In this case, we consider the correlated case with the presence of both channel/noise correlation. In this simulation setting, we generate the instances of $\mathbf{Y}$ according to case IV shown in Table II and we evaluate the knowledge of correlation on the PU SNR estimation performance using (21). To evaluate the knowledge of noise correlation only, we use case II of the table and to evaluate the knowledge of channel correlation only, we use case III. Similarly, to evaluate performance without knowledge of both channel/noise correlation, we use case I and to evaluate the knowledge of both channel/noise correlation, we use case IV of the table. From the figure, it can be noted that the performance is optimal assuming the knowledge of both channel/noise correlation. Moreover, the MSE performance with knowledge of noise correlation becomes better than with the knowledge of channel correlation at lower SNR values and the phenomenon reverses for higher SNR values $(>-1$ $\mathrm{dB}$ SNR in the figure). Therefore, it can be concluded that the PU SNR can be reliably estimated if we have the perfect knowledge of both channel/noise correlation at the CR sensing module.

Furthermore, to evaluate the performance of the proposed technique in case of the imperfect correlation model, we consider $10 \%$ static deviation in the considered value of the correlation coefficient for both the channel and noise correlation. Subsequently, we carry out SNR estimation based on the procedure mentioned in Section VI and evaluate the performance using (21) in terms of the normalized MSE versus SNR plot shown in Fig. 6. From the figure, it can be noted that the PU SNR can be estimated with less than $2.5 \%$ normalized MSE error up to the SNR value of $0 \mathrm{~dB}$ while considering $10 \%$ imperfect correlation knowledge. At the same value of SNR i.e., $0 \mathrm{~dB}$, the normalized MSE error is about $2.5 \%$ while considering perfect knowledge of both channel/noise correlation. Thus the normalized MSE performance degradation in case of $10 \%$ imperfect correlation knowledge is about $1.7 \%$ at the SNR value of $0 \mathrm{~dB}$. Moreover, it can be noted that this performance degradation increases for lower SNR values and decreases for higher SNR values following performance of the perfect correlation knowledge case beyond the SNR value of $3 \mathrm{~dB}$.

Figure 7 shows the normalized MSE versus SNR for considered cases with the simulation parameters $(\beta=1$, $\mathrm{SCN}=4$ and $N=100)$. In this simulation setting, we generate instances of $\mathbf{Y}$ according to the considered case and we evaluate the MSE performance with (21) by considering the same case from the table. From the figure, it can be noted 
TABLE II: Lookup table for SNR estimation

\begin{tabular}{|c|c|c|c|c|c|c|}
\hline $\mathrm{SCN}$ & $\beta$ & SNR (dB) & $\begin{array}{c}\text { Case I } \\
\lambda_{\max }\left(\mathbf{H} \mathbf{S}_{d}+\mathbf{Z}\right) \\
\end{array}$ & $\begin{array}{c}\text { Case II } \\
\lambda_{\max }\left(\mathbf{H} \mathbf{S}_{d}+\hat{\mathbf{Z}}\right)\end{array}$ & $\begin{array}{c}\text { Case III } \\
\lambda_{\max }\left(\hat{\mathbf{H}} \mathbf{S}_{d}+\mathbf{Z}\right)\end{array}$ & $\begin{array}{c}\text { Case IV } \\
\lambda_{\max }\left(\hat{\mathbf{H}} \mathbf{S}_{d}+\hat{\mathbf{Z}}\right)\end{array}$ \\
\hline 2 & 1 & 4 & 10.77 & 10.78 & 11.03 & 11.15 \\
\hline 2 & 1 & 2 & 7.45 & 7.47 & 7.58 & 7.73 \\
\hline 2 & 1 & 0 & 5.59 & 5.65 & 5.65 & 5.82 \\
\hline 2 & 1 & -2 & 4.70 & 4.79 & 4.71 & 4.88 \\
\hline 2 & 1 & -4 & 4.29 & 4.38 & 4.29 & 4.43 \\
\hline 2 & 1 & -6 & 4.08 & 4.19 & 4.08 & 4.21 \\
\hline 2 & 1 & -8 & 3.96 & 4.07 & 3.96 & 4.09 \\
\hline 2 & 1 & -10 & 3.90 & 4.01 & 3.90 & 4.01 \\
\hline 3 & 1 & 4 & 10.77 & 10.82 & 11.45 & 11.77 \\
\hline 3 & 1 & 2 & 7.45 & 7.52 & 7.82 & 8.19 \\
\hline 3 & 1 & 0 & 5.59 & 5.75 & 5.75 & 6.18 \\
\hline 3 & 1 & -2 & 4.70 & 4.93 & 4.75 & 5.17 \\
\hline 3 & 1 & -4 & 4.29 & 4.55 & 4.30 & 4.68 \\
\hline 3 & 1 & -6 & 4.08 & 4.35 & 4.08 & 4.42 \\
\hline 3 & 1 & -8 & 3.96 & 4.24 & 3.97 & 4.29 \\
\hline 3 & 1 & -10 & 3.90 & 4.18 & 3.90 & 4.20 \\
\hline 4 & 1 & 4 & 10.77 & 10.83 & 11.86 & 12.38 \\
\hline 4 & 1 & 2 & 7.45 & 7.57 & 8.05 & 8.65 \\
\hline 4 & 1 & 0 & 5.59 & 5.86 & 5.86 & 6.55 \\
\hline 4 & 1 & -2 & 4.70 & 5.08 & 4.78 & 5.45 \\
\hline 4 & 1 & -4 & 4.29 & 4.72 & 4.31 & 4.92 \\
\hline 4 & 1 & -6 & 4.08 & 4.52 & 4.08 & 4.63 \\
\hline 4 & 1 & -8 & 3.96 & 4.42 & 3.97 & 4.48 \\
\hline 4 & 1 & -10 & 3.90 & 4.35 & 3.90 & 4.39 \\
\hline
\end{tabular}

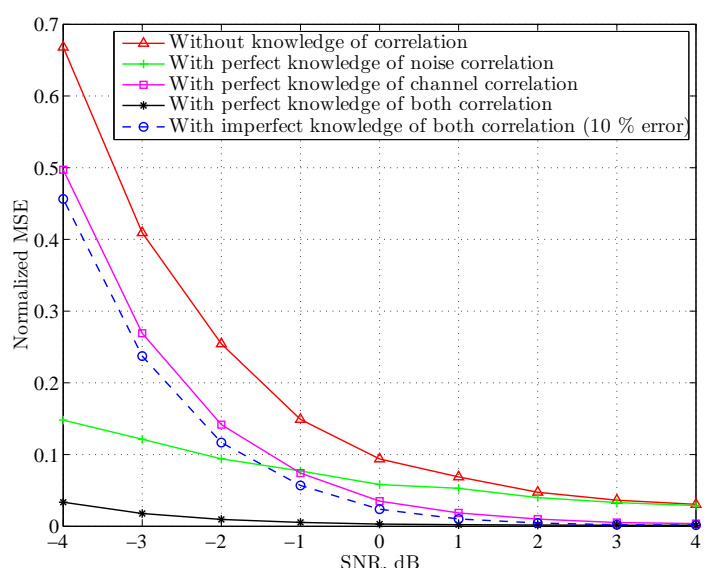

Fig. 5: Normalized MSE versus SNR with and without knowledge of channel/noise correlation $(\beta=1, \mathrm{SCN}=4, N=100)$

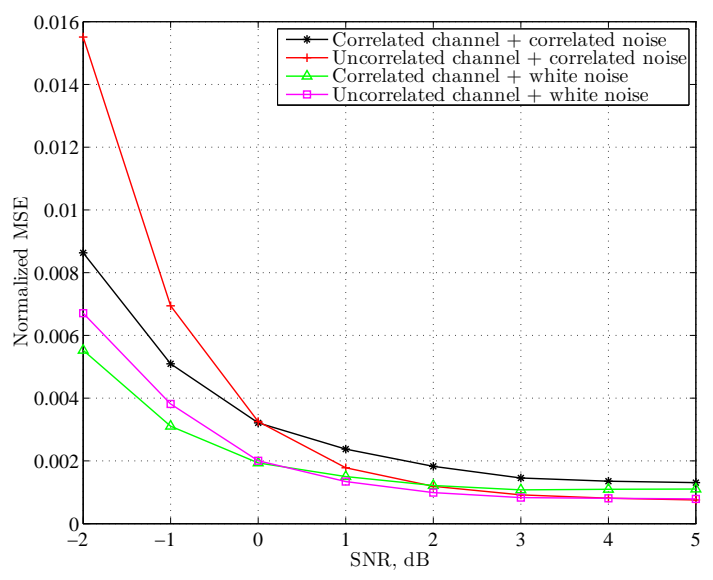

Fig. 6: Normalized MSE versus SNR for different cases $(\beta=1, \mathrm{SCN}=4, N=100)$

that the value of normalized MSE decreases with the increase in the SNR for all the cases. It can be noted that the SNR for all the cases can be reliably estimated with almost 0.1 $\%$ normalized MSE after $3 \mathrm{~dB}$. Furthermore, SNR for both channel uncorrelated and correlated cases can be estimated with $0.2 \%$ normalized MSE after SNR value of $0 \mathrm{~dB}$ at SCN value of 4 . For the case of presence of both channel/noise correlation, SNR can be estimated with $0.9 \%$ normalized MSE at $-2 \mathrm{~dB}$ and with $0.5 \%$ normalized MSE at $-1 \mathrm{~dB}$. Moreover, it can be noted that at lower SNR values, noise correlated case has higher normalized MSE than other cases and at higher values of SNR, noise correlated case provides better MSE performance than channel correlated case and both correlated case.

\section{CONCLUSION}

In this paper, spectrum sensing and SNR estimation problems in the presence of channel/noise correlation have been considered in the context of a CR. The performance of eigenvalue-based SS techniques has been studied in the presence of noise/channel correlation. It has been noted that noise correlation degrades the performance and channel correlation enhances the performance of the SCN-based SS techniques. Furthermore, theoretical expressions for a.e.p.d.f of the received signal's covariance matrix have been derived under signal plus noise hypothesis in the presence of channel correlation and in the presence of both channel/noise correlation. Moreover, an SNR estimation technique based on the maximum eigenvalue of the received signal's covariance matrix has been presented in order to estimate the PU SNR in the presence of both channel/noise correlation. The performance of the proposed technique has been evaluated in terms of normalized MSE. It can be concluded that the PU SNR can be reliably estimated using the proposed technique when the CR sensing module is aware of the channel/noise correlation.

\section{ACKNOWLEDGEMENT}

This work was supported by the National Research Fund, Luxembourg under AFR (Aids Training-Research) grant for $\mathrm{PhD}$ project (Reference 3069102) and the CORE project "CO2SAT: Cooperative and Cognitive Architectures for Satellite Networks". The authors would like to thank anonymous reviewers for providing their valuable suggestions. 
APPENDIX A

Random Matrix Theory Preliminaries

Let $F_{\mathbf{X}}(x)$ be the eigenvalue probability density function of a matrix $\mathbf{X}$.

Theorem 8.1: The Stieltjes transform $\mathcal{S}_{\mathbf{X}}(z)$ of a positive semidefinite matrix $\mathbf{X}$ is defined by [8]:

$$
\mathcal{S}_{\mathbf{X}}(z)=\mathbb{E}\left[\frac{1}{\mathbf{X}-z}\right]=\int_{-\infty}^{\infty} \frac{1}{\lambda-z} d F_{\mathbf{X}}(\lambda) .
$$

Theorem 8.2: The $\mathrm{R}$ transform is related to the inverse of Stieltjes transform as [8]:

$$
\mathcal{R}_{\mathbf{X}}(z)=\mathcal{S}_{\mathbf{X}}^{-1}(-z)-\frac{1}{z}
$$

Theorem 8.3: For a Wishart random matrix $\mathbf{X}$, the $\mathrm{R}$ transform of the density of eigenvalues of $\mathbf{X}$ is defined as [8]:

$$
\mathcal{R}_{\mathbf{X}}(z)=\frac{\beta}{1-z} .
$$

For any $a>0$,

$$
\mathcal{R}_{a \mathbf{X}}=a \mathcal{R}_{\mathbf{X}}(a z)
$$

Theorem 8.4: For a Wishart random matrix $\mathbf{X}$, the $\Sigma$ transform of the density of eigenvalues of $\mathbf{X}$ is defined as [8]:

$$
\Sigma_{\mathbf{X}}(z)=\frac{1}{z+\beta} \text {. }
$$

Theorem 8.5: The $\Sigma$ transform of the density of eigenvalues of $\mathbf{X}$ is related to the Stieltjes transform by the following relation [8]:

$$
\Sigma_{\mathbf{X}}(z)=-\frac{1+z}{z} \eta_{\mathbf{X}}^{-1}(1+z)
$$

Theorem 8.6: The $\eta$ transform of the density of eigenvalues of $\mathbf{X}$ is related to the Stieltjes transform by the following relation [8]:

$$
\eta_{\mathbf{X}}(z)=\frac{\mathcal{S}_{\mathbf{X}}\left(-\frac{1}{z}\right)}{z}
$$

\section{APPENDIX B}

\section{Proof of Theorem 6.3}

Assuming that signal and noise are uncorrelated to each other, for very large value of $N$, the following approximation can be written for correlated channel and uncorrelated noise scenario $[5]$.

$$
\lim _{N \rightarrow \infty} \hat{\mathbf{R}}_{\mathbf{Y}}(N) \approx p \hat{\mathbf{H}} \hat{\mathbf{H}}^{H}+\hat{\mathbf{R}}_{\mathbf{Z}}(N)
$$

The Stieltjes transform of $\hat{\mathbf{R}}_{\hat{\mathbf{H}}}=\frac{1}{N} \hat{\mathbf{H}} \hat{\mathbf{H}}^{H}$ can be written as [24]:

$\mathcal{S}_{\hat{\mathbf{R}}_{\hat{\mathbf{H}}}}(z)=\frac{z+2 z \mu+1-\beta+\sqrt{[z-(1+\beta)]^{2}-4 \beta(1+\mu z)}}{2 z(1+\mu z)}$.

The $\mathrm{R}$ transform of $\hat{\mathbf{R}}_{\hat{\mathbf{H}}}$ can be found by using (23) and $\mathrm{R}$ transform of $p \hat{\mathbf{R}}_{\hat{\mathbf{H}}}$ can be found by using (25) and can be written as:

$$
\mathcal{R}_{p \hat{\mathbf{R}}_{\hat{\mathbf{H}}}}(z)=-\frac{p}{2} \frac{\left(p z-1+\sqrt{\left(p^{2} z^{2}-2 p z+1-4 \mu \beta p z\right)}\right)}{\mu p z} .
$$

Then the combined $\mathrm{R}$ transform for $\hat{\mathbf{R}}_{\mathbf{Y}}$ can be written as:

$$
\mathcal{R}_{\hat{\mathbf{R}}_{\mathbf{Y}}}(z)=-\frac{p}{2} \frac{\left(p z-1+\sqrt{\left(p^{2} z^{2}-2 p z+1-4 \mu \beta p z\right)}\right)}{\mu p z}+\frac{\beta}{(1-z)}
$$

The inverse Stieltjes transform can be obtained by applying (32) on (23) and then the Stieltjes transform can be obtained by solving the quartic polymonial given by (19).

\section{APPENDIX C}

\section{Proof of Theorem 6.4}

Using the similar arguments as in Appendix B, the following approximation can be written for the case of presence of both channel/noise correlation.

$$
\lim _{N \rightarrow \infty} \hat{\mathbf{R}}_{\mathbf{Y}}(N) \approx p \hat{\mathbf{H}} \hat{\mathbf{H}}^{H}+\hat{\mathbf{R}}_{\hat{\mathbf{Z}}}(N) \text {. }
$$

The Stieltjes transform of $\hat{\mathbf{R}}_{\hat{\mathbf{Z}}}$ and $\hat{\mathbf{R}}_{\hat{\mathbf{H}}}$ are same and can be written as [24]:

$\mathcal{S}_{\hat{\mathbf{R}}_{\hat{\mathbf{H}}}}(z)=\mathcal{S}_{\hat{\mathbf{R}}_{\hat{\mathbf{z}}}}(z)=\frac{z+2 z \mu+1-\beta+\sqrt{[z-(1+\beta)]^{2}-4 \beta(1+\mu z)}}{2 z(1+\mu z)}$

The $\mathrm{R}$ transforms of $\hat{\mathbf{R}}_{\hat{\mathbf{H}}}$ and $\hat{\mathbf{R}}_{\hat{\mathbf{Z}}}$ can be found by using (23) and (34). The matrices $\hat{\mathbf{R}}_{\hat{\mathbf{H}}}$ and $\hat{\mathbf{R}}_{\hat{\mathbf{Z}}}$ are independent and any of these matrices can be written using eigenvalue decomposition as $\mathbf{U} \boldsymbol{\Lambda} \mathbf{U}^{H}$, where the elements of $\boldsymbol{\Lambda}$ are the eigenvalues which are distributed according to Theorem 5.1 with compact supports. As a result, $\hat{\mathbf{R}}_{\hat{\mathbf{H}}}$ and $\hat{\mathbf{R}}_{\hat{\mathbf{Z}}}$ are unitarily invariant and asymptotically free. Then the $\mathrm{R}$ transform of $p \hat{\mathbf{R}}_{\hat{\mathbf{H}}}$ can then be found by using (25) and can be written as:

$$
\mathcal{R}_{p \hat{\mathbf{R}}_{\hat{\mathbf{H}}}}(z)=-\frac{p}{2} \frac{\left(p z-1+\sqrt{\left(p^{2} z^{2}-2 p z+1-4 \mu \beta p z\right)}\right)}{\mu p z} .
$$

Then the combined $\mathrm{R}$ transform for $\hat{\mathbf{R}}_{\mathbf{Y}}$ can be written as:

$$
\begin{aligned}
\mathcal{R}_{\hat{\mathbf{R}}_{\mathbf{Y}}}(z)=-\frac{p}{2} & \frac{\left(p z-1+\sqrt{\left(p^{2} z^{2}-2 p z+1-4 \mu \beta p z\right)}\right)}{\mu p z} \\
& -\frac{1}{2} \frac{\left(z-1+\sqrt{\left(z^{2}-2 z+1-4 \mu \beta z\right)}\right)}{\mu z} .
\end{aligned}
$$

The inverse Stieltjes transform can be obtained by applying (36) on (23) and then the Stieltjes transform can be obtained by solving the quartic polymonial given by (20).

\section{REFERENCES}

[1] A. Goldsmith, S. Jafar, I. Maric, and S. Srinivasa, "Breaking spectrum gridlock with cognitive radios: An information theoretic perspective," Proc. IEEE, vol. 97, no. 5, pp. 894 -914, May 2009.

[2] S. K. Sharma, S. Chatzinotas, and B. Ottersten, "Satellite cognitive communications: Interference modeling and techniques selection," in 6th ASMS/SPSC Conf., Sept. 2012, pp. 111-118.

[3] T. Yucek and H. Arslan, "A survey of spectrum sensing algorithms for cognitive radio applications," IEEE Commun. Surveys Tutorials, vol. 11, no. 1 , pp. $116-130$, quarter 2009

[4] R. Tandra and A. Sahai, "SNR walls for signal detection," IEEE J. Sel. Topics Signal Process., vol. 2, no. 1, pp. 4 -17, Feb. 2008.

[5] Y. Zeng and Y. chang Liang, "Eigenvalue-based spectrum sensing algorithms for cognitive radio," IEEE Trans. Commun., vol. 57, no. 6, pp. $1784-1793$, June 2009.

[6] W. Zhang, G. Abreu, M. Inamori, and Y. Sanada, "Spectrum sensing algorithms via finite random matrices," IEEE Trans. Commun., vol. 60, no. 1, pp. $164-175$, Jan. 2012.

[7] A. Kortun, T. Ratnarajah, M. Sellathurai, C. Zhong, and C. Papadias, "On the performance of eigenvalue-based cooperative spectrum sensing for cognitive radio," IEEE J. Sel. Topics Signal Process., vol. 5, no. 1, pp. $49-55$, Feb. 2011.

[8] A. M. Tulino and S. Verdu, "Random matrix theory and wireless communications," Foundations and Trends Commun. Info. Theory, vol. 1, no. 1, pp. 1-182, 2004.

[9] R. Couillet and M. Debbah, Random Matrix Methods for Wireless Communications, 1st ed. Cambridge University Press, 2011. 
[10] P. Wang, J. Fang, N. Han, and H. Li, "Multiantenna-assisted spectrum sensing for cognitive radio," IEEE Trans. Veh. Technol., vol. 59, no. 4, pp. $1791-1800$, May 2010

[11] D.-S. Shiu, G. Foschini, M. Gans, and J. Kahn, "Fading correlation and its effect on the capacity of multielement antenna systems," IEEE Trans. Commun., vol. 48, no. 3, pp. 502 -513, Mar. 2000.

[12] H. Hui, "Influence of antenna characteristics on MIMO systems with compact monopole arrays," IEEE Antennas and Wireless Propag. Letters, vol. 8, pp. $133-136,2009$.

[13] K. Hassan, R. Gautier, I. Dayoub, E. Radoi, and M. Berbineau, "Predicted eigenvalue threshold based spectrum sensing with correlated multiple-antennas," in IEEE 75th Veh. Technol. Conf. (VTC Spring), May 2012, pp. $1-5$

[14] M. Matthaiou, M. Mckay, P. Smith, and J. Nossek, "On the condition number distribution of complex wishart matrices," IEEE Trans. Commun., vol. 58, no. 6, pp. $1705-1717$, June 2010.

[15] R. Couillet, J. Silverstein, Z. Bai, and M. Debbah, "Eigen-inference for energy estimation of multiple sources," IEEE Trans. Info. Th., vol. 57, no. 4, pp. 2420-2439, 2011.

[16] M. Vu, N. Devroye, and V. Tarokh, "On the primary exclusive region of cognitive networks," IEEE Trans. Wireless Commun., vol. 8, no. 7, pp. $3380-3385$, July 2009.

[17] T. Yucek, and H. Arslan, "MMSE Noise Plus Interference Power Estimation in Adaptive OFDM Systems," IEEE Trans. Veh. Technol., vol.56, no.6, pp.3857-3863, Nov. 2007.

[18] M. Fujii and Y. Watanabe, "A study on SNR estimation for cognitive radio," in IEEE Int. Conf. Ultra-Wideband, Sept. 2012, pp. $11-15$.

[19] T. Tian, H. Iwai, and H. Sasaoka, "Pseudo BER based SNR estimation for energy detection scheme in cognitive radio," in IEEE 75th Veh. Technol. Conf., May 2012, pp. 1 -5.

[20] W. Ejaz, N. Hasan, and H. S. Kim, "SNR-based adaptive spectrum sensing for cognitive radio networks," International J. Innovative Computing, Info. Control, vol. 8, no. 9, pp. 6095-6105, 2012.

[21] V. Chavali and C. da Silva, "Collaborative spectrum sensing based on a new SNR estimation and energy combining method," IEEE Trans. Veh. Technol., vol. 60, no. 8, pp. 4024-4029, 2011.

[22] S. K. Sharma, S. Chatzinotas, and B. Ottersten, "Eigenvalue based sensing and SNR estimation for cognitive radio in presence of noise correlation," IEEE Trans. Veh. Technol., vol. 62, no. 8, pp. 1-14, 2013.

[23] S. Chatzinotas, M. Imran, and R. Hoshyar, "On the multicell processing capacity of the cellular MIMO uplink channel in correlated Rayleigh fading environment," IEEE Trans. Wireless Commun., vol. 8, no. 7, pp. $3704-3715$, July 2009.

[24] X. Mestre, J. Fonollosa, and A. Pages-Zamora, "Capacity of MIMO channels: asymptotic evaluation under correlated fading," IEEE J. Sel. Areas Commun., vol. 21, no. 5, pp. 829 - 838, June 2003.

[25] L. Cardoso, M. Debbah, P. Bianchi, and J. Najim, "Cooperative spectrum sensing using random matrix theory," in 3rd Int. Symp. Wireless Pervasive Computing, May 2008, pp. $334-338$.

[26] F. Penna, R. Garello, and M. Spirito, "Cooperative spectrum sensing based on the limiting eigenvalue ratio distribution in Wishart matrices," IEEE Commun. Letters, vol. 13, no. 7, pp. 507 -509, July 2009.

[27] G. de Abreu, W. Zhang, and Y. Sanada, "Spectrum sensing algorithms via finite random matrix theory," in IEEE Int. Conf. Commun., June 2011, pp. $1-5$.

[28] Y. Zhou and F. Tian, "A spectrum sensing algorithm based on random matrix theory in cognitive radio networks," in 7th Int. Symp. Wireless Commun. Systems, Sept. 2010, pp. $971-975$.

[29] C. Zhong, M. McKay, T. Ratnarajah, and K.-K. Wong, "Distribution of the demmel condition number of Wishart matrices," IEEE Trans. Commun., vol. 59, no. 5, pp. 1309 -1320, May 2011.

[30] A. Kortun, M. Sellathurai, T. Ratnarajah, and C. Zhong, "Distribution of the ratio of the largest eigenvalue to the trace of complex Wishart matrices," IEEE Trans. Signal Process., vol. 60, no. 10, pp. 5527 -5532, Oct. 2012

[31] L. Wang, B. Zheng, J. Cui, S. Tang, and H. Dou, "Cooperative spectrum sensing using free probability theory," in IEEE Global Telecommun. Conf., Dec. 2009, pp. $1-5$.

[32] L. Wang, B. Zheng, J. Cui, and Q. Meng, "Cooperative MIMO spectrum sensing using free probability theory," in 5th Int. Conf. Wireless Commun., Networking and Mobile Computing, Sept. 2009, pp. $1-4$

[33] S. Kim, J. Lee, H. Wang, and D. Hong, "Sensing performance of energy detector with correlated multiple antennas," IEEE Signal Process. Letters, vol. 16, no. 8, pp. 671 -674, Aug. 2009.

[34] V. Banjade, N. Rajatheva, and C. Tellambura, "Performance analysis of energy detection with multiple correlated antenna cognitive radio in
Nakagami-m fading," IEEE Commun. Letters,, vol. 16, no. 4, pp. 502 -505 , April 2012

[35] L. Luo, P. Zhang, G. Zhang, and J. Qin, "Spectrum sensing for cognitive radio networks with correlated multiple antennas," Electronics Letters, vol. 47, no. 23, pp. $1297-1298,2011$.

[36] S. K. Sharma, S. Chatzinotas, and B. Ottersten, "The effect of noise correlation on fractional sampling based spectrum sensing," in IEEE Int. Conf. Commun., June 2013, pp. 1182-1187.

[37] S. Chatzinotas, S. K. Sharma, and B. Ottersten, "Asymptotic analysis of eigenvalue-based blind spectrum sensing techniques," in IEEE Int. Conf. Acoustics, Speech, and Signal Process., May 2013, pp. $4464-4468$.

[38] R. Janaswamy, "Effect of element mutual coupling on the capacity of fixed length linear arrays," IEEE Antennas and Wireless Propagation Letters, vol. 1, no. 1, pp. $157-160,2002$.

[39] S. Haykin, Communication Systems, 4th ed. John Wiley \& Sons, Inc.

[40] P. Bianchi, M. Debbah, M. Maida, and J. Najim, "Performance of statistical tests for single-source detection using random matrix theory," IEEE Trans. Info. Th., vol. 57, no. 4, pp. 2400-2419, 2011.

[41] B. Nadler, F. Penna, and R. Garello, "Performance of eigenvalue-based signal detectors with known and unknown noise level," in IEEE Int. Conf. Commun., 2011, pp. 1-5.

[42] Y.-C. Chen and Y.-T. Su, "MIMO channel estimation in correlated fading environments," IEEE Trans. Wireless Commun., vol. 9, no. 3, pp. 11081119,2010

[43] O. Tirkkonen and L. Wei, Foundation of Cognitive Radio Systems: Exact and asymptotic analysis of largest eigenvalue based spectrum sensing. InTech, 2012, no. 978-953-51-0268-7, ch. 1.

[44] D. Voiculescu, "Multiplication of certain non-commuting random variables," J. Operator Theory, vol. 18, pp. 223-235, 1987.

[45] - "Addition of certain non-commuting random variables," J. Funct. Analysis, vol. 66, pp. 323-346, 1986.

[46] S. M. Kay, Fundamentals of Statistical Signal Processing: Detection Theory. Prentice Hall, 1998, vol. II.

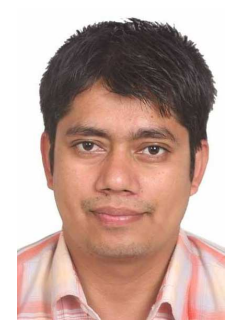

Shree Krishna Sharma (B.E.,M.A.,M.Sc.,M. Res.) received the B.E. degree in electronics and communication from Birla Institute of Technology, Mesra, India, in 2004; the M.Sc. degree in information and communication engineering from the Institute of Engineering, Pulchowk, Nepal, in 2010; the M.A. degree in economics from Tribhuvan University Patan Multiple Campus, Patan, Nepal, and the M.Res. degree in computing science from Staffordshire University, Staffordshire, U.K., in 2011. He is currently working toward the Ph.D. degree with the Interdisciplinary Centre for Security, Reliability and Trust, University of Luxembourg, Luxembourg City, Luxembourg.

In the past, he was also involved with Kathmandu University, Dhulikhel, Nepal, as a Teaching Assistant, and he served as a Part-Time Lecturer for eight engineering colleges in Nepal. He was with Nepal Telecom for more than four years as a Telecom Engineer in the field of information technology and telecommunication. His research interests include cognitive wireless communications, resource allocation, and interference mitigation in heterogeneous wireless networks. Mr. Sharma received an Indian Embassy Scholarship for his B.E. study, an Erasmus Mundus Scholarship for his M.Res. study, and an Aids Training-Research Ph.D. grant from the National Research Fund of Luxembourg. 


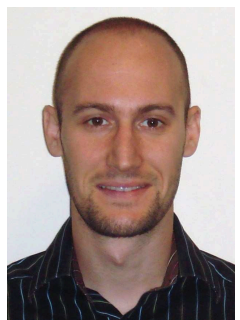

Symeon Chatzinotas (S'06-M'09) received the M.Eng. degree in telecommunications from Aristotle University of Thessaloniki, Thessaloniki, Greece, in 2003 and the M.Sc. and the Ph.D. degrees in electronic engineering from the University of Surrey, Surrey, U.K., in 2009.

In the past, he has worked on numerous research and development projects for the Institute of Informatics and Telecommunications, National Center for Scientific Research Demokritos, Athens, Greece; the Institute of Telematics and Informatics, Center of Research and Technology Hellas, Thessaloniki, Greece; and the Mobile Communications Research Group, Center of Communication Systems Research, University of Surrey. He is currently a Research Scientist with the Interdisciplinary Centre for Security, Reliability and Trust, University of Luxembourg, Luxembourg City, Luxembourg. He is the author of more than 60 technical papers in refereed international journals, conferences, and scientific books. His research interests include multiuser information theory, cooperative and cognitive communications, and transceiver optimization for terrestrial and satellite networks.

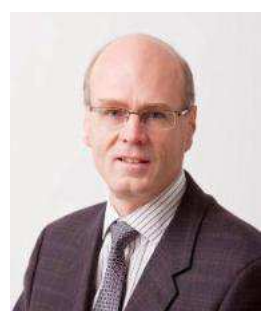

Björn Ottesten (S'87-M'89-SM'99-F'04) was born in Stockholm, Sweden, in 1961. He received the M.S. degree in electrical engineering and applied physics from Linköping University, Linköping, Sweden, in 1986 and the Ph.D. degree in electrical engineering from Stanford University, Stanford, CA, in 1989.

He has held research positions with the Department of Electrical Engineering, Linkping University; the Information Systems Laboratory, Stanford University; and the Katholieke Universiteit Leuven, Leuven, Belgium. In 1991, he was appointed as a Professor of signal processing with the Royal Institute of Technology (KTH), Stockholm, where he was the Head of the Department for Signals, Sensors, and Systems from 1992 to 2004 and the Dean of the School of Electrical Engineering from 2004 to 2008. From 1996 to 1997, he was the Director of Research with ArrayComm Inc., San Jose, CA, which is a start-up company based on his patented technology. Since 2009, he has been the Director of the Interdisciplinary Centre for Security, Reliability and Trust, University of Luxembourg, Luxembourg City, Luxembourg. His research interests include wireless communications, stochastic signal processing, sensor array processing, and time-series analysis. Dr. Ottersten has served as an Associate Editor for the IEEE TRANSACTIONS ON SIGNAL PROCESSING and on the Editorial Board of the IEEE Signal Processing Magazine. He is currently the Editor-in-Chief of the EURASIP Signal Processing Journal and a member of the Editorial Board of the EURASIP Journal of Advances Signal Processing. He has coauthored papers that received IEEE Signal Processing Society Best Paper Awards in 1993, 2001, and 2006, as well as three IEEE conference papers that received Best Paper Awards. In 2011, he received the IEEE Signal Processing Society Technical Achievement Award. He is one of the first recipients of the European Research Council advanced research grant. 